\title{
Design and Implementation of University Network
}

\author{
Mugdha Sharma, Chirag Pupreja, Akash Arora
}

\begin{abstract}
Information technology is being used at numerous places fulfilling various purposes. Doing work at one place by professors and sharing it at another place either to faculty members and students becomes quite difficult. As doing tasks on one PC, a professor might have to visit different classes and labs due to various reasons. Similarly a student working on a project might have to access a particular project document in multiple labs and classes. Head of Department has to visit various rooms and classes in a particular day. It would be very tedious job to carry laptop everywhere. There might be a situation when a user wants to share a particular data with more than 1 user. There can also be a situation when a particular message/ information need to be shared with the entire university. So this research work proposes a novel approach to communicate among various users that are present at different sites at the same time where at university premises network system is being proposed which would help departments to share information among faculty members and students Proposed approach takes the help of sharing common network domain by DNS and applies heterogeneous BUS topology model to explore various concepts like topology design, creating dynamic host configuration protocol, sub net masking, DNS and VLAN within a single network with the help of Cisco Packet Tracer to make the network more secured and cost effective.
\end{abstract}

Index Terms: Cisco Packet Tracer (CPT), Dynamic Host Configuration Protocol (DHCP), Domain name System (DNS), Virtual LAN (VLAN), Personal Computer (PC).

\section{INTRODUCTION}

A computer network is defined as a co-lateral connection of various devices that are connected through different communication medium/channels. Main purpose of a computer network is to facilitate the users to communicate among themselves and share resources with other users. Networks can be classified into various categories with different characteristics that are mentioned and explained further in this section of the research work.

\section{A. Cisco Packet Tracer (CPT)}

CPT handles multiple tasks at once and comprehensive network simulation that help in proto- typing of a network, teaching and learning software developed by Cisco Networking Academy to perform and analyze various network activities and offers a unique combination of

Revised Manuscript Received on July 22, 2019.

Mugdha Sharma, Department of Computer Science Engineering, Bhagwan Parshuram Institute of Technology, IP University, Delhi, India.

Chirag Pupreja, Department of Computer Science Engineering, Bhagwan Parshuram Institute of Technology, IP University, Delhi, India.

Akash Arora, Department of Computer Science Engineering, Bhagwan Parshuram Institute of Technology, IP University, Delhi, India. realistic simulation and visualization experiences, tedious assessment and different opportunities for multiuser collaboration. It enables a user to create and build a network by selecting optimum devices based on their characteristics, usage of various topologies which help in choosing the best topology, creating DHCP server, using different troubleshooting commands for different configurations. It also enables the user to build a network for different labs, classroom, hostels for a university or an organization. Before implementing any network in real life, organizations and colleges should build their network using Cisco Packet Tracer which helps in giving an overview of the functionalities and different cost effective ways to implement that network in real life.

\section{B. Switch}

A switch is a device that sends data in the form of packets from one user to another user by looking at the physical device address i.e. MAC address or Media Access Control. It determines to which MAC address the packet belong to and if the packet belong to none of the port then it sends the packet to all the ports. A switch has an advantage to function as Router as well.

\section{Hub}

A hub is a simplest networking device which connects various network devices to each other through ethernet. Multiple devices are connected to hub as it consist of various input and output ports. All the devices are set on a same network segment to transmit data from one device to the other.

\section{Local Area Network (LAN)}

LAN is used to connect various computers available in a limited geographical area with the help of physical wires. LAN provides a high-bandwidth communication and that too through a medium which is not expensive at all [1]. In today's digital world, network should be easily accessible all the time from anywhere which also offers fast, secure, and reliable services to the users [2]. Performance of LAN depends on external factors also such as physical devices/ systems should be capable enough to sustain the network and provide reliable and secured performance. There are various professional tools available in the market that can design and maintain LANs [3] which are known as simulation tools and can predict the performance of a LAN as per various factors such as hardware, topology, traffic load or the purpose of the application.

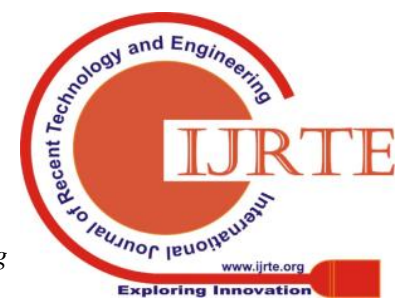




\section{E. Virtual Local Area Networks (VLANs)}

A Computer Network can be created by partitioning a single layer network into multiple distinct broadcast domains, which will be isolated mutually and packets can only pass between them with the help of routers.

This type of network domain is known as a Virtual Local Area Network.

\section{F. Access Control List}

A sequential series of commands that instructs a router to accept or deny the different types of packets based on specific criteria is known as an access control list. These are usually applied on the router's interfaces to make it more secure network. A router is programmed to validate each and every packet based on the conditions that are specified in the ACL and then filter outs the packets.

\section{LITERATURE REVIEW}

The main objective of this research work is to study the existing work done in the domain of computer networks, wired and wireless both. There are various existing network infrastructure but those are mainly on wired network and that too for small labs. The current network infrastructure contains the connection that is sufficient for laboratory and that too with the help of hubs. The proposed network design will be useful for an entire university and every user of the network will be interlinked with each other. With the help of Cisco Packet Tracer, students of the university can practice and experiment on the proposed network design. Cisco Networking Academy Program (CNAP) introduced Packet Tracer as a tool for teaching and learning of computer network course by providing -simulation, visualization, authoring, collaboration capabilities and assessment\| which made it possible to visualize a network connection of an entire university feasible [5]. With the help of Cisco Packet Tracer, students can built their own network topologies in a visual way by dragging, placing, connecting, and clustering various virtual network devices such as switches, hubs, routers, LAN, VLAN, VOIP, WIFI, workstations and servers [6]. According to current proposed network model there are network of university which uses ring topology but here BUS topology is used[8]. And the optical fibre used is for a ring topology in earlier work but here with the help of BUS topology an effective and cost effective network is been laid for the whole university.[9] Also the existing network creates different network to be established for different labs but here the whole university works on a single network which includes various facilities such as transmitting messages, accessing data on any physical computer within the network, accessing same speed in various geographical areas within university network. This way the network will be able to provide the facility of VOIP i.e. voice over Internet Protocol which will allow the users within the same network to communicate to each other with the help of internet. Security is also the main concern in this university network. Packet Tracer is chosen because of its easy installation (Windows and Linux) [5]. Packet Tracer allows users experience more interactive and similar to real life using different networking devices in a user friendly simulation environment [7].

\section{METHODOLOGY}

Network access for the hostels is implemented through a separate switch being implemented for the boy's hostel, and similarly for the girl's hostel. A separate switch is implemented for each of the hostels (for example, 3 switches for three boys' hostels) to ensure that the students of one hostel cannot access the Internet services of another hostel. This reduces system traffic. Unlimited network access is arranged for the Director's Office and with a reasonably high speed. The Director's Office will not have any sites blocked, but will be provided the same speed by the ISP even after fair usage limit.

This proposal is extremely simple to implement and is pretty useful so that users can have all the access on a single click. Network access for all the departments other than CSE, one PC is shown as the - HOD PC\| - the one specifically reserved for the Head of the Dept. All other PCs are collectively shown as -Other\|. For CSE, due to the number of labs, multiple switches are created; each switch provides access to one lab where devices in each lab are connected to a VLAN. Unlimited network access for the staff is provided and is implemented by assuming that although the staff will not get their speeds reduced after the fair usage limit, there will be still some restricted sites (the same sites restricted for the students) that are accessible to the Director only. Network access for the library is managed by simply attaching a hub to one of the main routers to enable Internet access. Network access for the campus is managed with the help of $\mathrm{Wi}-\mathrm{Fi}$ access instead of a wired connection, so a DSL modem is a must for providing this facility. Network access for the guest house is managed by simply attaching a switch to one of the main routers to enable Internet access.

Instant network access is provided to all the users on the network. Portal for login is not been added as it will be cumbersome. Instead, two Wi-Fi routers are added. One is for the guests (open access, 1/3rd the normal speed), and the other for the students, staff, etc. (password protected WPA2/PSK, normal speed). For security purposes, a selective example is taken to showcase the security measures that can be taken in the future - for example, we have enabled a 256-bit password in the console for the router. The Internet Service Provider provides speed at a rate of $10 \mathrm{MB} / \mathrm{s}$. Network Administrator is the person(s) who can decrease, increase or otherwise modify the speeds of the network, shutdown or restart the entire network (only in cases of severe eventualities), add more sub-networks to the main networks (example - adding more users to a VLAN, in case a new department is established).It is also possible for the routers to get their firmware replaced, updated, etc. by the network administrator(s). There may be other features added (or deleted) in the future which will be updated as necessary.

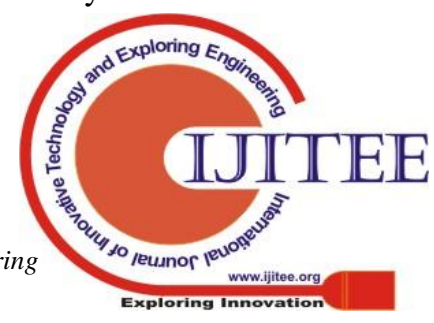




\section{A. Security}

Different tools and techniques have been used to make the network more secure and robust. With the help of VLAN the system becomes more secure and threat free.

The usage of ACL (Access Control List) makes it more secure by permitting the access of server to certain users and keep the access away from hostel. The server files will not be accessed by the hostel premises which make the network more secure and reliable.

The hostel students will be able to ping the server but will not be allowed to access them due to ACL.

Apart from this feature, MAC flooding attack is also controlled and prevented with the help of port security. In this method, the switches are configured to limit the number of MAC addresses that can be learned on ports connected to end stations. Also for security purposes, a password is been set up on switches which will allow only certain users to access the domain. Access List is always used to ensure the security of the Result Server, i.e. to prevent unnecessary access to the files.

\section{B. Cost Effective}

To make the proposed system cost effective VLANs have been used instead of using routers everywhere. The VLAN will help in reducing the cost of different devices. Also multiple systems are connected to same switch to make the network more effective. The VLAN are helpful in establishing the network connection only when it is required by the user.

\section{RESULTS}

The proposed design of the university network is been established in a simulated environment. The routers and the computer system were configured accordingly. Packets were sent from one computer to the other and the transmission of such data to their destination was successful in an efficient manner. Through the network, emails and files can be sent and received by staff and students of the institution. In order to provide security to the network various methods are implemented. Password protection is also applied on the switches for restricted usage. The authentication is done within the network. The ACL allows only those files to flow from the server that are allowed for the students. With addition to this VLANs are installed for the better security and protection of the network. Also MAC flooding attack is also prevented within the simulated network. Domain name is set as akash@bpit.com which will have the access to different users within the university network from any system. This will also help in broadcasting the messages within the network at the same time. The simulated network was tested and it performed just like a real computer network. This shows that university network will work perfectly when implemented in various other environment and scenarios.

\section{A. Conclusion}

The proposed approach helps in designing the network outlook for the university network that produces the different functionalities within a single network such as security of the network, wireless area network, mobility as well as restricting the access to limited users, operational efficiencies and a cost effective network. The template provided by this paper is applicable for any network, provided it is not too large. The network is fully featured with ISP, network administration, interconnection of various departments, VLANs for connection of Department Heads and wardens, network access for library using hubs; guesthouse has an open Wi-Fi too. This paper describes the network design scenario approved by Cisco, as well as where we can apply these scenario within the various locations of a university network. Finally, key network foundation services such as switching, routing, multicast, and high availability are given for the university network scenario in an efficient way.

\section{B. Figures and Tables}

Following table describes the comparison between a Hub and a Switch.

\section{TABLE I. COMPARISION BETWEEN HUB AND SWITCH}

\begin{tabular}{|c|c|c|c|}
\hline \multirow{2}{*}{$\begin{array}{c}\text { Serial } \\
\text { No. }\end{array}$} & \multicolumn{3}{|c|}{ Comparison } \\
\cline { 2 - 4 } & $\begin{array}{c}\text { Area of } \\
\text { Comparison }\end{array}$ & HUB & Switch \\
\hline 1. & Cost & Expensive & Cost Effective \\
\hline 2. & $\begin{array}{c}\text { Transmission } \\
\text { Mode }\end{array}$ & Half Duplex & Full Duplex \\
\hline 3. & MAC Address & $\begin{array}{c}\text { Cannot store } \\
\text { MAC address }\end{array}$ & $\begin{array}{c}\text { Can store MAC } \\
\text { address in a lookup } \\
\text { table }\end{array}$ \\
\hline 4. & Security & $\begin{array}{c}\text { No configuration } \\
\text { can be done }\end{array}$ & $\begin{array}{c}\text { More secure as MAC } \\
\text { flooding be avoided } \\
\text { through port security }\end{array}$ \\
\hline 5. & Cast & Unicast & Multicast \\
\hline 6. & Device Type & Passive Device & Active Device \\
\hline 7. & Collision & $\begin{array}{c}\text { Occur during } \\
\text { setup }\end{array}$ & Does not occur \\
\hline 8. & Layer & $\begin{array}{c}\text { Operates at } \\
\text { Physical Layer }\end{array}$ & $\begin{array}{c}\text { Operates at Data } \\
\text { Link Layer }\end{array}$ \\
\hline
\end{tabular}

Following figures explains the architecture and processing of proposed system.

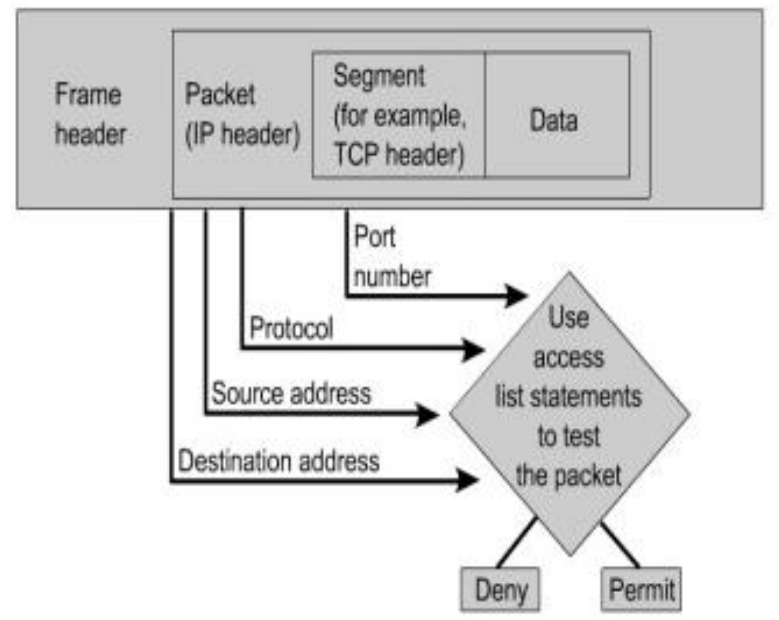

Fig. 1. Access List Decision Base

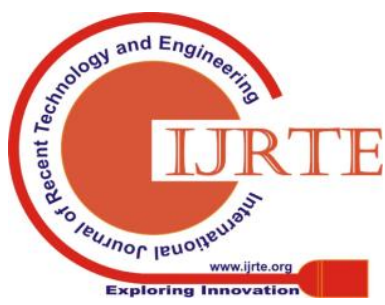




\section{Design and Implementation of University Network}

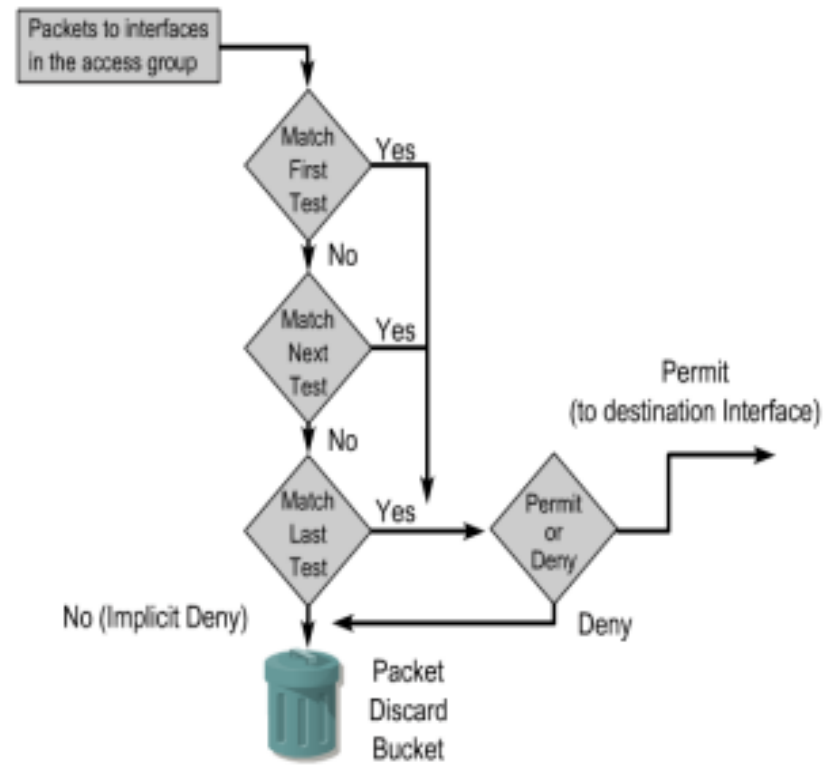

Fig. 2. Access List Filter

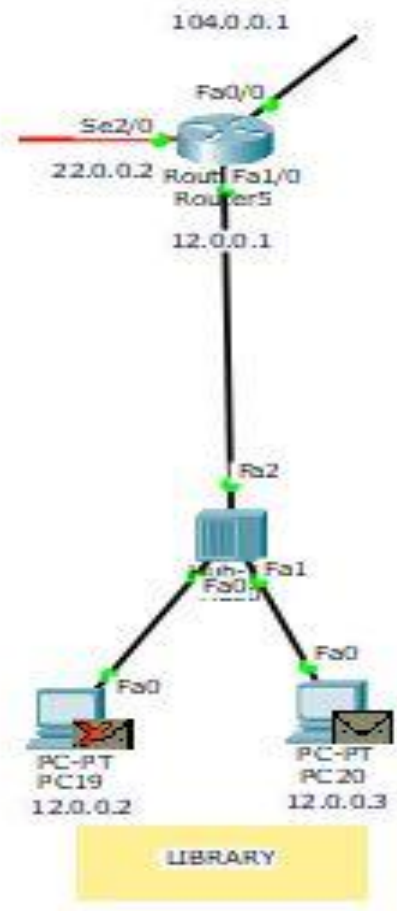

Fig. 3. Hub

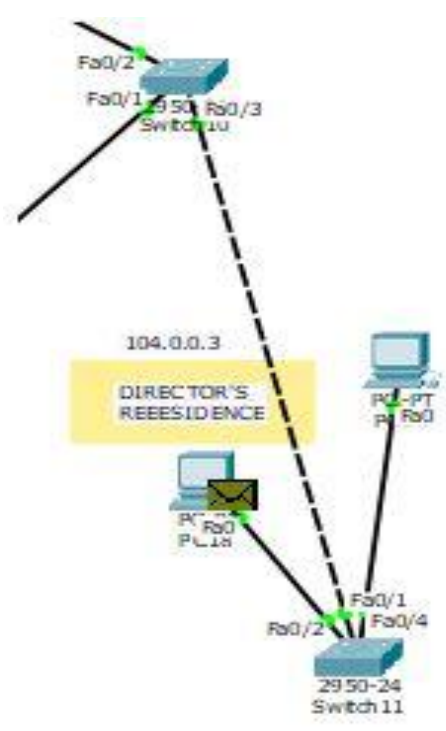

Fig. 4. Switch

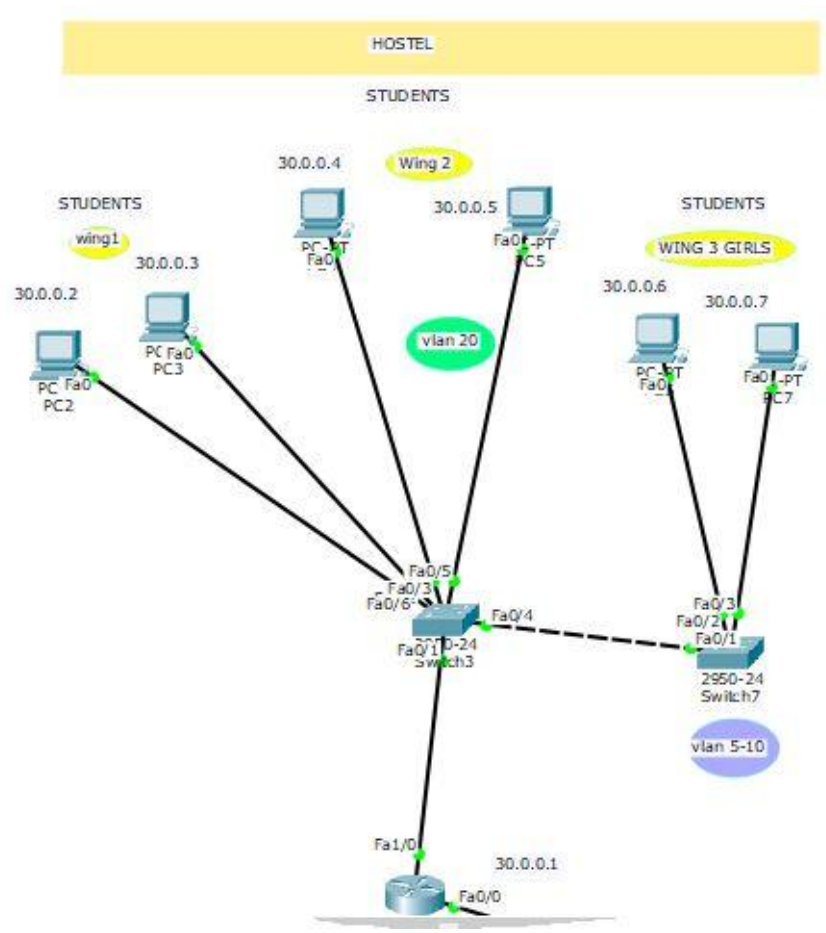

Fig. 5. Access Control List in a University Network

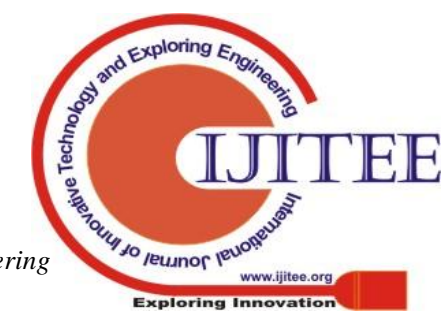




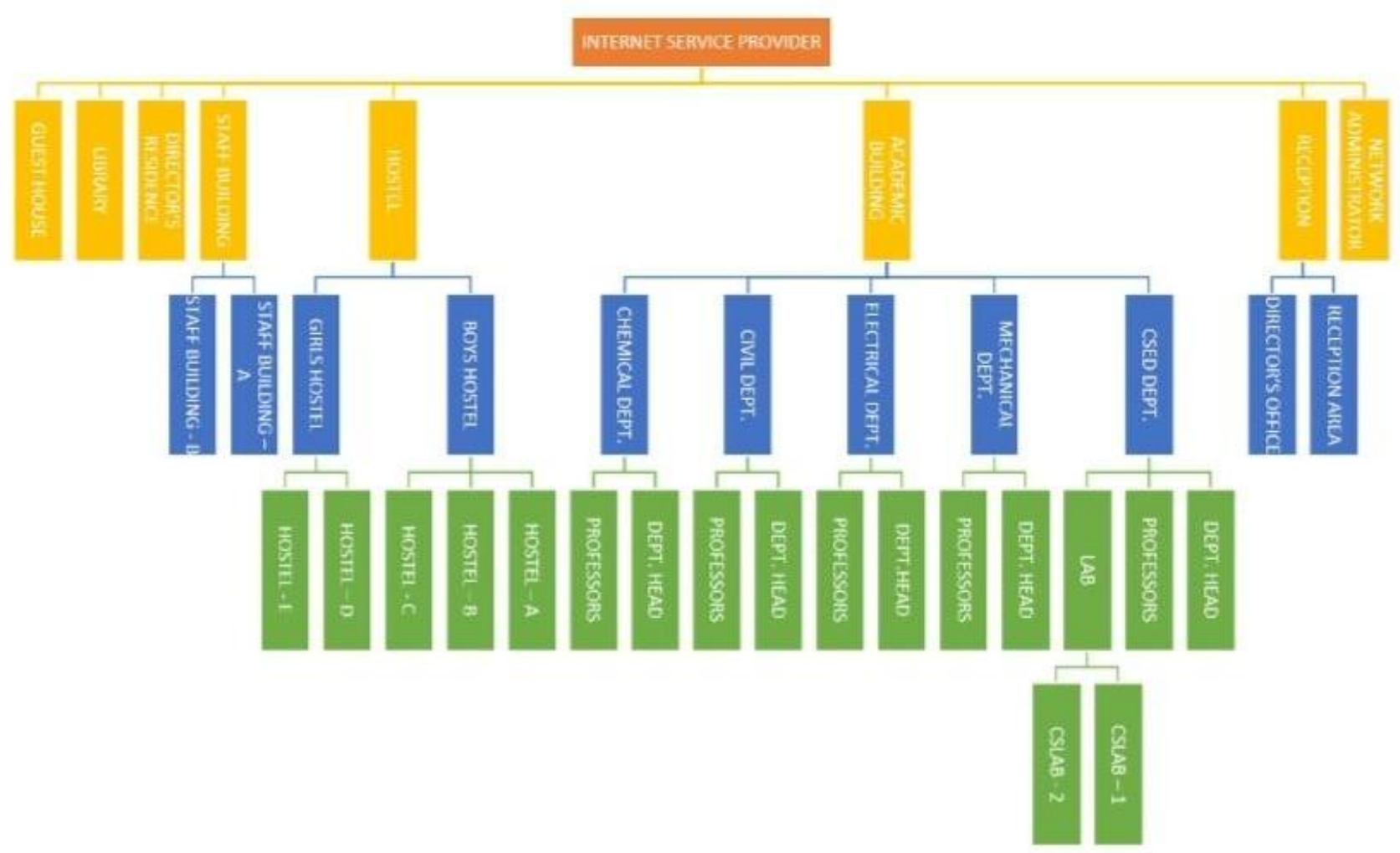

Fig. 6. Basic Architecture of University

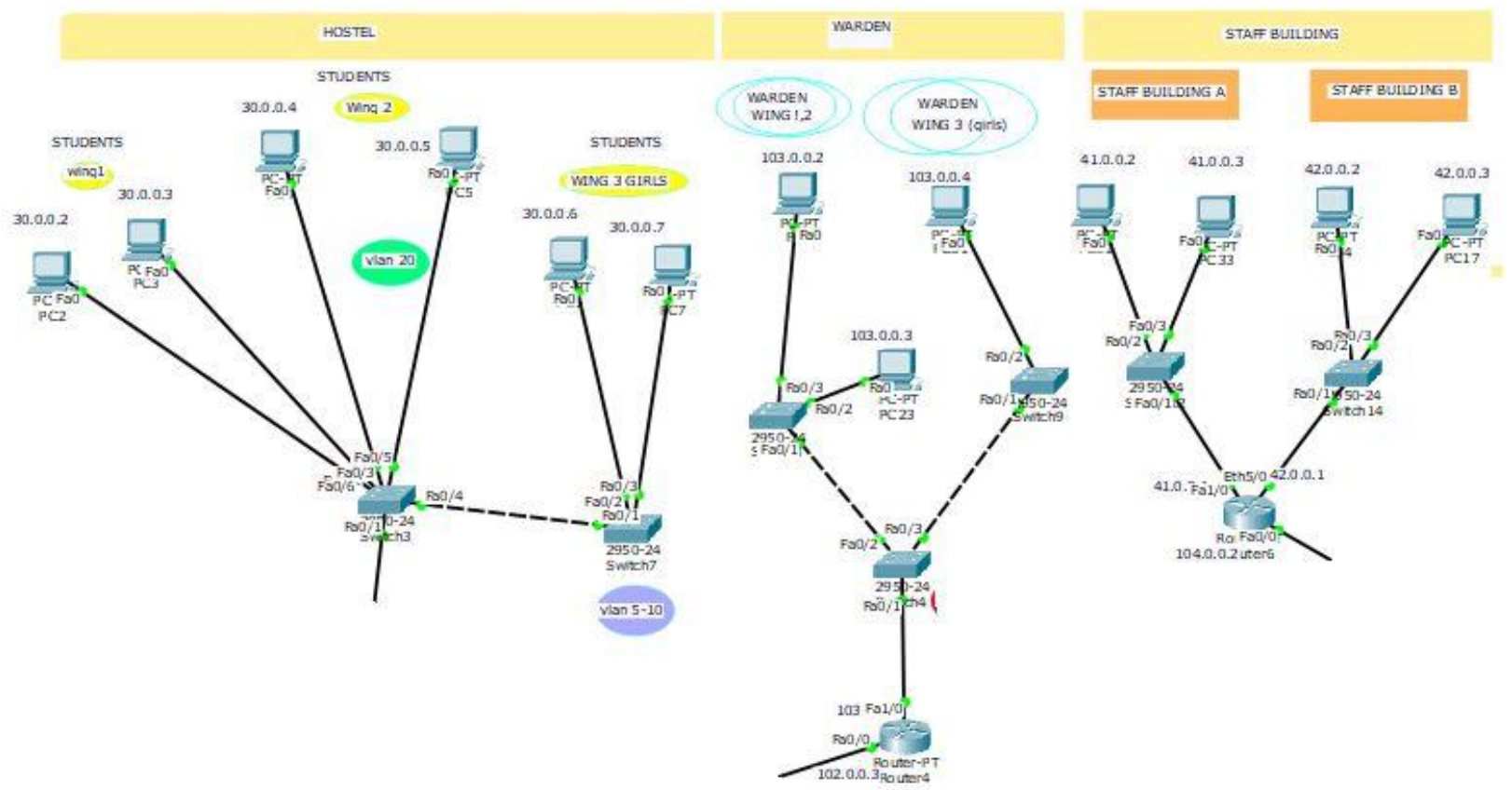

Fig. 7. Cost Effectiveness by Installing VLANs.

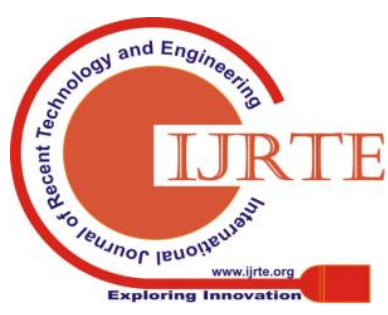




\section{Design and Implementation of University Network}

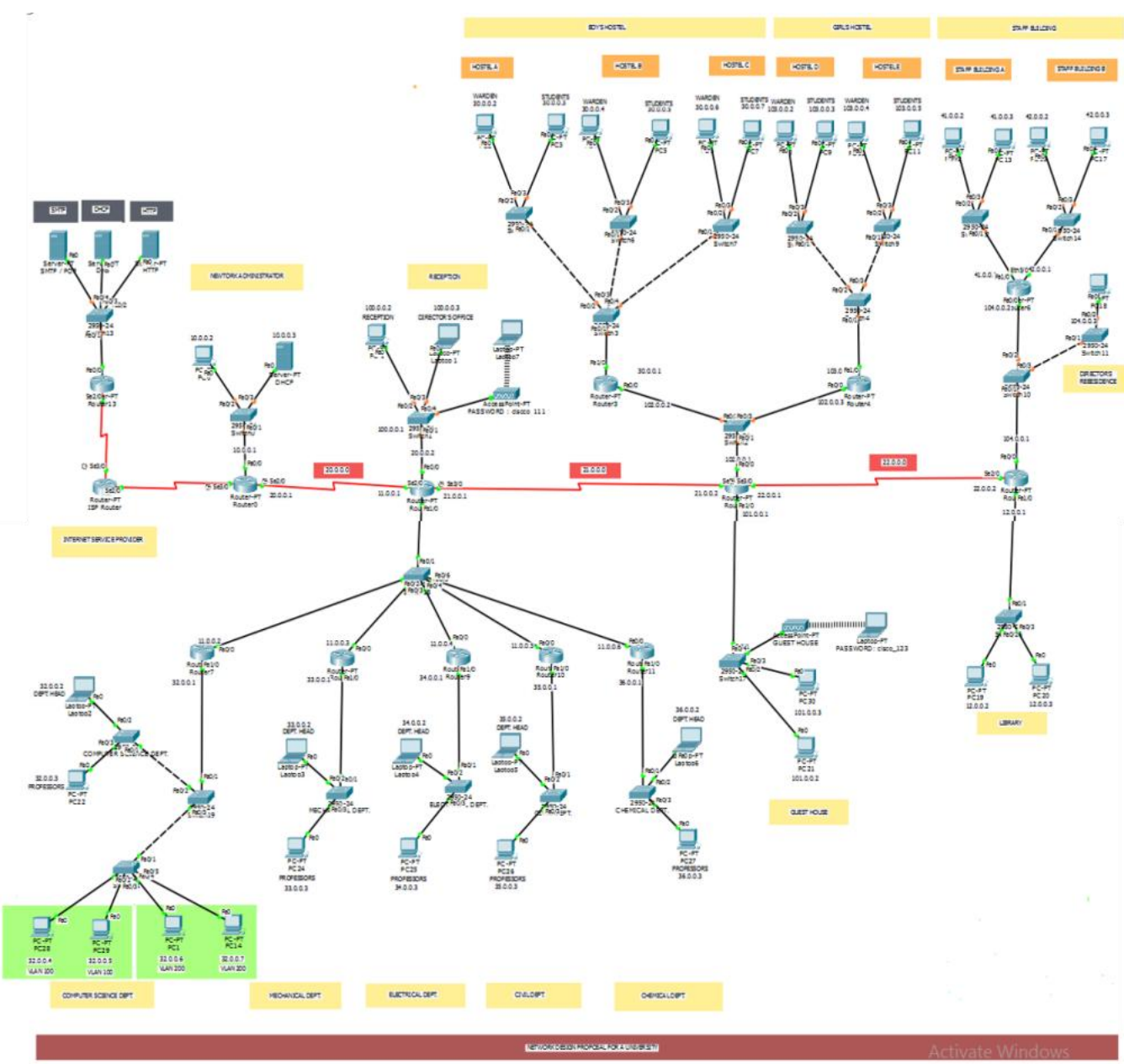

Fig. 8. Complete University Network.

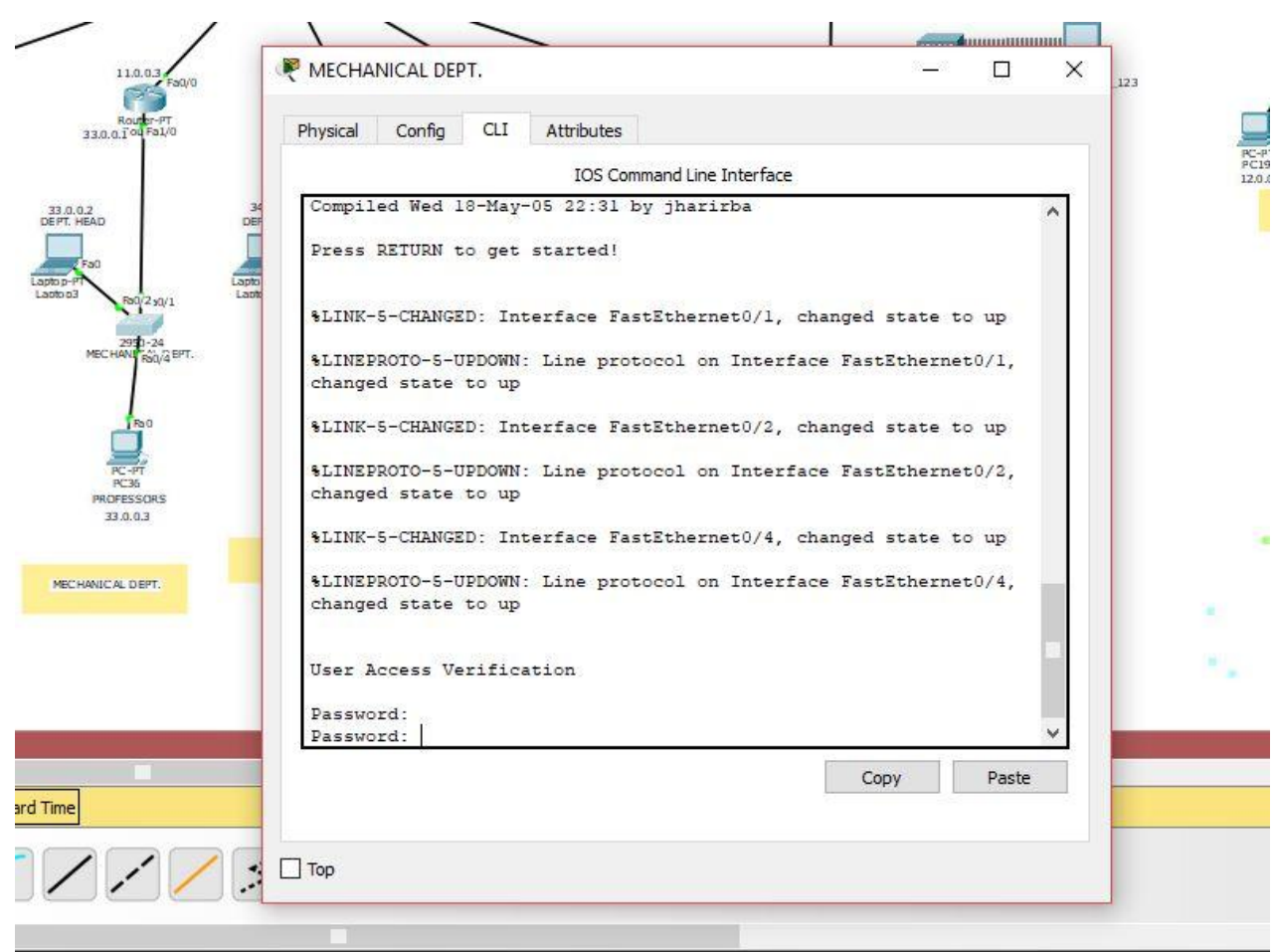

Fig. 9. Password Protection in Switch. 


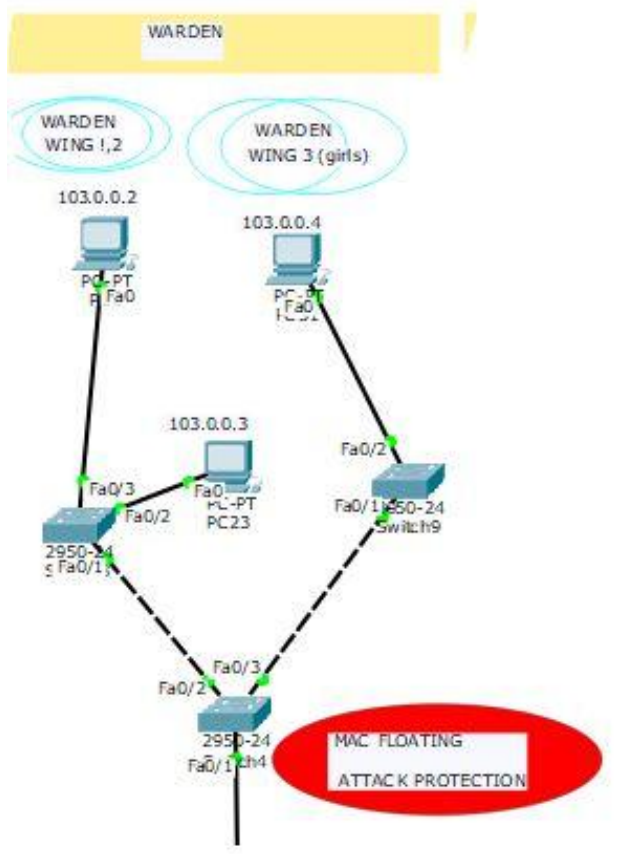

Fig. 10. MAC Flooding Attack Protection in Switch.

\section{ACKNOWLEDGMENT}

Authors express their deep sense of gratitude to the Principal of Bhagwan Parshuram Institute of Technology, Prof. Payal Pahwa for her keen interest in promoting research in the institute and have always been an inspiration for achieving great heights.

\section{REFERENCES}

1. Tim Reardon, Planning, Designing and operating local area networks, DISAM Journal, Summer, 1997.

2. www.wikipedia.org/wiki/computer_networks, Retrieved 10th October, 2016.

3. www.wikipedia.org/wiki/local_area_network, Retrieved 10th October, 2016.

4. W. Buchanan,-Correlation between academic and skills-based tests in computer networks,\| Br. J. Educ. Technol., vol. 37, no. 1, pp. 69- 78, 2006

5. A. K. Adesemowo and M. Gerber, -E-skilling on fundamental ICT networking concepts - Overcoming the resource constraints at a South African University, $\|$ in Proc. e-Skills for Knowledge Production and Innovation Conference, pp. 1-16,2014.

6. J. Expósito, V. Trujillo, and E. Gamess, -Using visual educational tools for the teaching and learning of EIGRP,\| in Proc. the World Congress on Engineering and Computer Science, vol. I, pp. 169-174, 2010.

7. J. Janitor, F. Jakab, and K. Kniewald, -Visual learning tools for teaching/learning computer networks: Cisco networking academy and packet tracer,\| in Proc. 2010 Sixth International Conference on Networking and Services, pp. 351-355, 2010.

8. International Journal of Advanced Computer Research (ISSN (print) 2249-7277 ISSN (online): 2277-7970) Volume-4 Number-3 Issue-16 September-2014 -Plan, Design and Simulation of University Network\|.

9. International Journal of Mechanical \& Mechatronics Engineering IJMME-IJENS Vol:12 No:06 -University Optical Fibre Network Access Optimisation: A Case Studyll

\section{AUTHORS PROFILE}

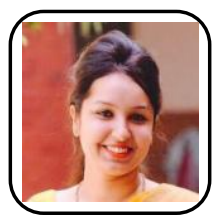

Mugdha Sharma completed Bachelor of Technology and Master of Technology from Guru Gobind Singh Indraprastha University of New Delhi, India in year 2014. She is currently working as Assistant Professor in Department of Computer Science, Guru Gobind Singh Indraprastha University of New Delhi, India since 2014 She has 5 years of teaching experience and more than 7 years of Research Experience.

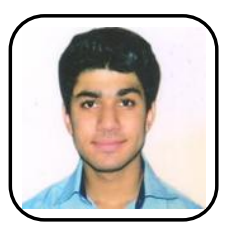

Chirag Pupreja is a final year student of Computer Science Engineering, Guru Gobind Singh Indraprastha University of New Delhi. His main research work focuses on Networking, Data Mining, Big Data Analytics, and Computational Intelligence based systems.

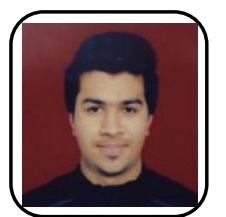

\section{Akash Arora}

is a final year student of Computer Science Engineering, Guru Gobind Singh Indraprastha University of New Delhi. His main research work focuses on Networking, Data Mining, Big Data Analytics, and Computational Intelligence based systems. 